2012-04-30

\title{
An ERP investigation of regional and foreign accent processing
}

Goslin, Jeremy

http://hdl.handle.net/10026.1/9954

10.1016/j.bandl.2012.04.017

Brain and Language

Elsevier BV

All content in PEARL is protected by copyright law. Author manuscripts are made available in accordance with publisher policies. Please cite only the published version using the details provided on the item record or document. In the absence of an open licence (e.g. Creative Commons), permissions for further reuse of content should be sought from the publisher or author. 


\title{
An ERP investigation of regional and foreign accent processing
}

\author{
Jeremy Goslin*, Hester Duffy, Caroline Floccia \\ School of Psychology, University of Plymouth, UK
}

\section{A R T I C L E I N F O}

\section{Article history:}

Accepted 30 April 2012

Available online 12 June 2012

\section{Keywords:}

Language processing

Lexical access

Speech variability

Normalisation

ERP

Accents

PMN

N400

\begin{abstract}
A B S T R A C T
This study used event-related potentials (ERPs) to examine whether we employ the same normalisation mechanisms when processing words spoken with a regional accent or foreign accent. Our results showed that the Phonological Mapping Negativity (PMN) following the onset of the final word of sentences spoken with an unfamiliar regional accent was greater than for those produced in the listener's own accent, whilst PMN for foreign accented speech was reduced. Foreign accents also resulted in a reduction in N400 amplitude when compared to both unfamiliar regional accents and the listener's own accent, with no significant difference found between the $\mathrm{N} 400$ of the regional and home accents. These results suggest that regional accent related variations are normalised at the earliest stages of spoken word recognition, requiring less top-down lexical intervention than foreign accents.
\end{abstract}

(c) 2012 Elsevier Inc. All rights reserved.

\section{Introduction}

Unfamiliar regional or foreign accents in our native language are frequently encountered in everyday life and can cause difficulties in the comprehension of speech. Traditionally the study of accents and dialects has fallen within the scope of sociolinguistics, that is, the study of language variation and change. It is only recently that the mechanisms by which we process and represent accent and dialect-related variation has been of interest to psycholinguists (Adank, Evans, Stuart-Smith, \& Scott, 2009; Adank \& McQueen, 2007; Bradlow \& Bent, 2003; Clarke, 2000; Clarke \& Garrett, 2004; Clopper \& Bradlow, 2009; Clopper \& Pisoni, 2004a, 2004b; Derwing, Rossiter, Munro, \& Thomson, 2004; Dufour, Nguyen, \& Frauenfelder, 2007; Evans \& Iverson, 2004; Flege, 1988; Floccia, Butler, Goslin, \& Ellis, 2009; Floccia, Goslin, Girard, \& Konopczynski, 2006; Jongman, Wade, \& Sereno, 2003; Lane, 1963; Maye, Aslin, \& Tanenhaus, 2008; Munro \& Derwing, 1995a, 1995b; Schmid \& Yeni-Komshian, 1999; Sebastián-Gallés, VeraConstán, Larsson, Costa, \& Deco, 2009; Sumner \& Samuel, 2009). It is often acknowledged that accents can be ranked on a perceptual scale according to their acoustic distance from native speech (e.g. Clarke \& Garrett, 2004), with most foreign accents standing at the far end of the perceptual scale and regional accents somewhere in-between. Under this assumption, referred to here as the Perceptual Distance Hypothesis, the mechanisms underlying regional accent processing would simply be attenuated versions of

\footnotetext{
* Corresponding author. Address: School of Psychology, University of Plymouth, Drake Circus, Plymouth PL4 8AA, UK. Fax: +44 1752584808.

E-mail address: jeremy.goslin@plymouth.ac.uk (J. Goslin).
}

those activated during foreign accent processing. In favour of this hypotheses is the observation that spoken words are identified more slowly when they are produced with an unfamiliar accent as compared to participants' home accent (e.g. $30 \mathrm{~ms}$ in Floccia et al., 2006) and even more slowly when they are produced in a non-native accent (around $100 \mathrm{~ms}$ in Floccia et al., 2006).

However, based upon both linguistic observation and further behavioural evidence it has also been suggested that the quantitative differences between these delays could also mask functional differences in the processing and representation of non-native and regional accents (Adank et al., 2009; Chambers, 2002; Floccia et al., 2006, 2009; Girard, Floccia, \& Goslin, 2008). One observation that supports this Different Processes hypothesis is the interaction between the segmental and suprasegmental systems of the native and recently acquired non-native languages in foreign speakers (see Best, McRoberts, \& Goodell, 2001; Flege, 1988). The foreign accent that arises as a consequence of this interaction embodies variations that bear no relation to the native listener's maternal phonology, and as such would necessitate the activation of 'offline' rescue procedures. For instance, an English speaker listening to a French accent will often need to adapt to an incomplete stress system because of the difficulty French speakers usually have processing stress contrasts that are not lexically contrastive in their language (Dupoux, Pallier, Sebastian, \& Mehler, 1997). On the other hand, regional accents mainly consist of coherent deviations in phonetic, phonological, phonotactic and prosodic information found within the language (e.g. Wells, 1982). For example, Southern British English speakers produce the word "bath" with an advanced, long, low, tense, unrounded vowel /a:/, while Northern British English speakers tend to use the short, lax and unrounded 
vowel /æ/ (Wells, 1982). Both phonemes are found in Southern and Northern British English accents, therefore in order to adapt listeners simply have to learn the differences in the lexical distribution of these two vowels. The variability in the speech signal resulting from foreign phonology is likely to require more complex normalisation mechanisms than those involved in accounting for different lexical distributions within the existing native phonology.

Behavioural evidence supporting the Different Processes Hypothesis also comes from the developmental literature. At around five years of age both French (Girard et al., 2008) and British English speaking children (Floccia et al., 2009) have more difficulty categorising a regional accent than a foreign accent, even when the strength of accents are equivalently rated (Floccia et al., 2009). This suggests that the linguistic characteristics of a native unfamiliar accent are less perceptually salient to the children at this age. In addition, it is well established that when moving to a new region children are able to learn the new regional accent without problems (Chambers, 1992), whereas children do not seem to acquire non-native accents even when exposed to them (Chambers, 2002).

Another source of evidence indicating differential processing for foreign and regional accents comes from studies of accent adaptation in word identification (Clarke \& Garrett, 2004; Floccia et al., 2006; Munro \& Derwing, 1995a) that generally show much easier adaptation to foreign than regional accents (Adank \& McQueen, 2007; Clarke \& Garrett, 2004; Maye et al., 2008). For example, after a 20 min exposure to isolated words generated in an artificial regional accent in which all vowels were lowered, Maye et al. (2008) did not find any evidence for adaptation in lexical decision reaction times. Similarly, in an animacy decision task Adank and McQueen (2007) found that a strong delay in reaction times for isolated words produced in an unfamiliar accent was no different before and after a $20 \mathrm{~min}$ exposure to the accent. However, when Clarke and Garrett (2004) used a cross-modal matching test to compare the comprehension of native English speakers native accent and a foreign Spanish accent, they found that the initial impairment caused by the foreign accent had been fully adapted to within a few sentences of exposure (but see Floccia et al., 2009). Evans and Taylor (2010) reported stronger adaptation to foreign than regional accents when they were mixed with noise in a comprehension task, although participants were slower and made more mistakes with the foreign accents. However, it is possible that the greater adaptation shown for foreign accents could be because foreign accents are generally more difficult to process (e.g. Floccia et al., 2006), giving greater room for improvement. Finally, Pinet, Iverson, and Evans (2011) also found modest adaptation to both types of accent, this time using a repetition task.

As we have shown, both linguistic and empirical observation lends plausibility to the Different Processing Hypotheses, but how could this be integrated into current spoken word recognition models? In a classic abstract-entry model of word recognition (e.g. Marslen-Wilson, 1987; McClelland \& Elman, 1986; Norris, 1994; Pallier, Colomé, \& Sebastián-Gallés, 2001), it is reasonable to assume that accent normalisation mainly operates through low-level phonological mechanisms, that is, at a pre-lexical processing stage (see Samuel \& Kraljic, 2009 for a discussion; Sumner, 2011). Recent data on spoken word recognition also indicates top-down involvement of lexical representations in pre-lexical processing (Davis, Johnsrude, Hervais-Adelman, Taylor, \& McGettigan, 2005; Newman \& Connolly, 2009; Norris, McQueen, \& Cutler, 2003), with mismatch signals propagated down from lexical levels to tune pre-lexical accommodation of continued unfamiliar input. However, even with this intervention the normalisation still takes place at the pre-lexical level. Lexical representations are not modified; rather pre-lexical representations must be tuned to compensate for distortions or discrepancies in the signal. According to the
Perceptual Distance Hypothesis this would take place for both regional and foreign accents, with greater tuning required for the latter than the former. In contrast, the Different Processing Hypothesis would predict a different cognitive (and possibly neural) locus for regional and foreign accent normalisation. As regional accents predominantly involve language coherent phonological variations they could be dealt with primarily at the highly language-specific (e.g. Cutler, Mehler, Norris, \& Segui, 1986) pre-lexical level, whereas unfamiliar phonological phenomena that typify foreign accents would require greater involvement from top-down lexical levels. The potential for flexibility in the allocation of resources during word recognition has already been exemplified by a number of studies. In certain situations the normalisation of vocoded speech (Davis et al., 2005) or learning of ambiguous sounds (Eisner \& McQueen, 2005; Norris et al., 2003) would appear to be more robust when listeners can use lexical information. Whilst in others, such as the adaptation to time-compressed (Pallier, Sebastian-Gallés, Dupoux, Christophe, \& Mehler, 1998) and also for isolated vocoded words and non-words (see Hervais-Adelman, Davis, Johnsrude, \& Carlyon, 2008) normalisation would appear limited to pre-lexical phonological processing.

It would be impractical to evaluate the two hypotheses using the reaction-timed tasks used in previous studies as they only capture the end-point of speech processing, whilst the hypotheses make predictions specific to particular stages within this process. Instead we propose to compare the electrophysiological characteristics of regional and foreign accented speech perception, allowing us to examine normalisation and perceptual processes over the course of comprehension.

\subsection{Electrophysiological measures of accented speech}

Unfortunately, there have only been a few studies that have used electrophysiological methods to examine the processing of accented speech, and even these do not provide a consistent understanding of these processes. Berman, Mandelkern, Phan, and Zaidel (2003) recorded ERPs and PET blood flow during dichotic presentation of word pairs produced in Australian, British, French and Hindi accents. Neuroimaging data showed that word detection produced left lateralized activation in posterior frontal cortex and inferior temporal lobe, while accent detection produced homologous activation in the right hemisphere. Unfortunately the authors did not distinguish between the regional and foreign accents in their study, and nor did they attempt to link ERP with specific components (see Desroches, Newman, \& Joanisse, 2009).

Conrey, Potts, and Niedzielski (2005) compared the perception of vowel contrasts in two American accents, one of which merges the vowels in "pen" and "pin", and the other that does not. In a cross modal matching task participants first saw a visual representation of a sentence ending with a word containing a vowel of interest (e.g. pin, pen, tin, ten) and had to match that word with a spoken version that had either a matched or mismatched vowel. Behavioural data showed a clear improvement in matching performance for the unmerged vowel accent population (59\% vs $78 \%$ ). However, no clear accent effect was found in the Mismatch Negativity, which was surprising in light of previous studies that have found cross-linguistic differences in phonemic processing in MMN (Naatanen et al., 1997; Rivera-Gaxiola, Csibra, Johnson, \& Karmiloff-Smith, 2000), with the N400 epoch similarly unenlightening. However, vowel congruency was found to modulate the Late Positive Component (LPC) in the unmerged vowel accent population, but not the one where the vowels had merged. The authors interpreted this late component as showing effects of conscious phonological decision processes.

In a similar study Brunellière, Dufour, Nguyen, and Frauenfelder (2009) compared the perception of the French vowel contrast 
$|\varepsilon /-| e /$, which is no longer contrastive in the South of France but is in standard (northern) French, with a control contrast $\mid \varnothing /-/ y /$, which is present in all accents. Standard French speaking listeners making a forced discrimination of the vowel were found to be slower and less accurate with the merging $/ \varepsilon /-/ e /$ contrast than the control one. Further comparisons showed that the control vowels elicited differences across P200 (190-230), MMN (270-310), and a later time period (372-486 ms), whilst for the merging vowels there was only a difference in the MMN. It was suggested that this was due to the difficulty in distinguishing the merging vowels at a phonemic processing level due to participants' exposure to merging accents, leading to a change in the organisation of regions that selectively answer to native language vowels (Shestakova, Brattico, Soloviev, Klucharev, \& Huotilainen, 2004). This was supported in a follow-up study, where Brunellière, Dufour, and Nguyen (2011) found that the $|\varepsilon /-| e /$ contrast induced different cortical topographies only in the standard French population, but not in the Southern French population.

\subsection{The present study}

As we have seen, electrophysiological correlates to accent processing are far from being consistent, with the possibility of hemispheric asymmetry (Berman et al., 2003) and potential activity stretching from the MMN (Conrey et al., 2005) to the LPC (Berman et al., 2003). One problem with direct comparisons of prior studies is the difference in task and procedures adopted in each, and the difficulty in disentangling ERP related to perceptual processing of accents from relatively complex task specific effects. In this study we simply compare the ERP associated with the perception of fully formed sentences spoken in different accents. The only task required of the participants is to detect occasional sentences containing semantic probe words to ensure their attention remains focussed upon the speech stimuli. For the remaining stimuli ERP will be time-locked onto the final words of sentences spoken with either the participant's own native accent, one of two other native English accents, or one of two foreign accents. By presenting regional and foreign accents in spoken sentences, rather than segments or words with specific phonetic contrasts, we expose the listener to the full range of segmental and suprasegmental properties that characterise accents in natural speech. Thus we treat an accent as the sum of the whole of all the deviations from the listeners own accent, rather than focussing upon one specific aspect. This also dictates the use of sentences, rather than words or syllables, as shorter stimuli would not encapsulate many accent-related differences, and time-locking ERP to the final word to ensure prior exposure to both short and longer-term (such as stress) accent related speech changes. Using this task we will examine potential deviations in the two most researched ERP components likely to be relevant to accent related speech, namely the Phonological Mapping Negativity (PMN; Connolly, Phillips, Stewart, \& Brake, 1992; Newman, Connolly, \& McIvor, 2003) and the N400 (Kutas \& Hillyard, 1984).

The PMN peaks between 250 and $300 \mathrm{~ms}$ after the stimulus onset, distributed along a fronto-central line. It is thought to reflect pre-lexical processing or lexical selection as it is modulated by differences between the phonological candidates formed by contextual expectation and the incoming acoustic-phonetic information (Connolly \& Phillips, 1994; Desroches et al., 2009; Hagoort \& Brown, 2000). It is argued that it is dissociable from the N400 (e.g. Connolly \& Phillips, 1994; Desroches et al., 2009; Newman \& Connolly, 2009; Newman et al., 2003) as it is only sensitive to auditory stimuli and appears to be insensitive to the lexical status of mismatched stimuli (Newman \& Connolly, 2009). It has been further suggested (Newman \& Connolly, 2009) that this component provides a goodness-of-fit measure between the expected phonology and the actual acoustic-phonetic input. The Perceptual Distance Hypothesis would predict that the difference between stored 'home' phonological representations and accented speech would increase the amplitude of this negative wave, with an even greater magnitude for foreign accents reflecting the increased resources required by normalisation processes. The Different Processes Hypothesis would predict more involvement of pre-lexical processing for regional accent normalisation than for foreign accent normalisation (and inversely, more involvement at the lexical level for the foreign accent). This hypothesis would also predict increased PMN for the regional accents than the home accent, reflecting pre-lexical normalisation for regional accents (e.g. Newman \& Connolly, 2009). If we interpret this normalisation process as the means by which accent-related "noise" may be removed such that an abstract representation can be computed before access to the lexicon (e.g. Luce \& McLennan, 2005), then the failure of this process with foreign accents would leave this "noise" intact. In this case we might expect to see less pre-lexical activation for foreign accented speech translating to a reduction in PMN as compared to the home accent, as seen in ERP responses to low intelligible speech signals (e.g. Martin, Sigal, Kurtzberg, \& Stapells, 1997).

The N400 is a negativity distributed along the central and parietal areas that usually peaks at around $400 \mathrm{~ms}$ after stimulus onset and is sensitive to semantic or lexical incongruities (e.g. Desroches et al., 2009; Kutas \& Hillyard, 1984). Whether or not accent related differences would also emerge for the N400 component is a key question: if normalisation of accented speech is achieved at the pre-lexical processing level for both types of accents, then lexical activation or integration should not be affected by the different speech styles (see Sumner, 2011). If this were not the case then the Perceptual Distance Hypothesis would still predict quantitative deviations in the $\mathrm{N} 400$ relative to distance between the home accent and the regional and foreign accents. In contrast, the pre-lexical normalisation of regional accents postulated by the Different Processes Hypothesis would predict a similar N400 for regional and home accents (see Samuel \& Kraljic, 2009), deviating only for foreign accents.

Predictions for the direction of potential N400 deviations are unclear. If the amplitude of this component simply increased as the integration of lexical information becomes more difficult (e.g. Desroches et al., 2009) the Perceptual Distance Hypothesis would predict that the component would be greater for regional than home accents, and greater still for foreign accents. However, this is complicated by the findings of Aydelott, Dick, and Mills (2006) and Boulenger, Hoen, Jacquier, and Meunier (2011). In the former study sentences with semantically congruent or incongruent word endings were presented to participants intact, or low-pass filtered at $1 \mathrm{kHz}$ to reduce intelligibility. As expected, the $\mathrm{N} 400$ was larger for incongruent than congruent words, but the magnitude of this effect was found to be lower for distorted speech than clear speech. Aydelott et al. suggest that acoustic degradation reduces the availability of semantic information and thus the neural response of word context. This was also corroborated Boulenger et al.'s study, where participants heard sentences with high or low cloze probability final words that contained varying durations of signal reversal. They found that increasing the duration of the signal reversal reduced the N400 amplitude for low close probability (CP) sentences, but increased amplitude in high CP sentences. This was thought to be the result of the ambiguity caused by acoustic/phonetic distortion reducing incongruity in low CP sentences, but increasing it in high $\mathrm{CP}$ sentences. If we were to liken accent related variation to the artificially generated acoustic/phonetic distortion seen in these two studies, we would expect a reduction in the $\mathrm{N} 400$ relative to the amount of distortion caused by the accent. That is, with the low CP sentences used in the present study 
Table 1

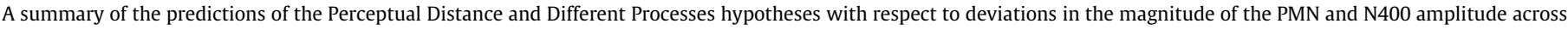
the three accents.

\begin{tabular}{|c|c|c|}
\hline & Perceptual Distance Hypothesis & Different Processes Hypothesis \\
\hline PMN & Foreign $>$ Regional $>$ Home & Regional $>$ Home $>$ Foreign \\
\hline N400 & Foreign $>$ Regional $>$ Home or Foreign $<$ Regional $<$ Home & Foreign $>$ Regional $=$ Home or Foreign $<$ Regional $=$ Home \\
\hline
\end{tabular}

the N400 amplitude would be greater in foreign than regionally accented sentences. Likewise, the Perceptual Distance Hypothesis would predict the N400 amplitude of regional accented sentences to be significantly less than those produced in the home accent. A summary of the predictions of the two hypotheses for PMN and N400 components can be seen in Table 1.

\section{Materials and methods}

\subsection{Participants}

Sixty students from the University of Plymouth (age range: 1840; mean: 20.2; 15 male) participated in this experiment in return for course credit. All of these participants were native speakers of English, born and raised in the South-West of England. All participants were right handed, and reported that they had normal hearing and no language or neurological impairments. Of these participants five were rejected because of equipment failure (2) or excessive EOG artifacts (3: more than 30\% contaminated segments in any experimental condition).

\subsection{Stimuli}

Spoken sentences were recorded from 12 male speakers originating from three British regions and two other countries. The four speakers in the 'Home' accent condition were born and raised in the South-West of England. Two speakers for the 'Regional' accent originated from South Wales, and the two others from Yorkshire (Leeds). Two speakers for the 'Foreign' accent were native to the north of Italy and two from central Poland, all having relocated to the UK within three years of the recordings. Each of the speakers was asked to read aloud from one of six lists of 55 unique sentences (making 330 unique sentences across the six lists). These lists were arranged to create two stimuli sets; set 'HYP' consisted of sentences spoken by two home accented speakers, two Yorkshire and two Polish speakers. Set 'HWI' consisted of sentences spoken by the remaining two home accented speakers, plus the two Welsh and two Italian speakers. In both sets the home accented speakers read from the same sub-set of sentences, whilst each sentence read by a regional accent speaker in 'HYP' was read by a foreign speaker in 'HWI', and vice versa. This ensured that any experimental differences between regional and foreign accents could not be due to the form of the sentences (as they were balanced).

All sentences were between 9 and 15 syllables in length, did not contain an embedded clause, and ended with a disyllabic trochaic noun. They were ambiguous as to whether the final word would contain the name of an animal, such as "Roger searched the church tower for the pastor", and as such had a low cloze probability. Each sentence was recorded in a soundproof room using an Audio Protechnica Pro 41 microphone then directly digitized at $44 \mathrm{kHz}$ with 16 bits per sample, and later normalised to the same mean amplitude level. The lexical characteristics of the final word of the sentences were matched across the five accents for the number of morphemes and phonemes, lexical frequency (subtitle frequency: Brysbaert \& New, 2009), phonological uniqueness, phonological neighbourhood, and phonological Levenshtein distance (PLD20) (all ps $<0.11$; see Table 2 for details). No attempt was made to control or adjust the temporal characteristics of the stimuli as this forms an inherent part of the differences between accents.

No significant difference was found between final word durations of the three accent categories (Home: $528.4 \mathrm{~ms}$, Regional: $517.2 \mathrm{~ms}$, Foreign: $525.7 \mathrm{~ms} ; F(2,376)<1$, see Table 2$)$, but differences in the duration of the preceding sentences were found (Home: $1852.4 \mathrm{~ms}$, Regional: $1902.8 \mathrm{~ms}$, Foreign: $2047.8 \mathrm{~ms}$; $F(2,376)=26.33, p<0.001)$.

In addition each speaker recorded 6-7 additional sentences that ended with an animal name ("His wife managed to win a bag of goldfish").

The identity and perceived strength of each accent were ascertained by presenting the stimulus sentences to a set of pre-test participants who had been raised in the South-West of England. Each of the 20 participants heard a different subset of 76 sentences that ranged across all of the twelve speakers, counterbalanced such that each sentence in the stimulus set would be rated by at least two participants. Sentences were presented over headphones (Sennheiser HD580) in random order using E-Prime Professional v2.0 (Psychology Software Tools Inc., Pittsburgh, PA). After hearing a sentence the participant was asked to identify it from a list of the five accents used in the experiment, with an additional choice for "I don't know". After this they were asked to rate their confidence in their decision, from 0 ("Not at all confident") to 5 ("Very confident"). Finally, they were asked to make a judgment on the strength of the accent, using Received Pronunciation as a baseline (0), on a scale from 0 ("Not at all strong") to 5 ("Very strong").

As can be seen in Table 3, the participants were found to correctly identify the accent in $64 \%$ of sentences, with the best performance for their own accent (82.9\% correct), whilst the Yorkshire accent was only correctly identified in $34.5 \%$ of cases, being misidentified as South-West in $53.6 \%$ of cases. It should also be noted that regional accents were rarely misidentified as foreign accents ( $2.7 \%$ of cases), and vice versa ( $4.6 \%$ of cases). When combined into the three accent categories, a one-way ANOVA found a significant effect of accent $(F(2,38)=24.62, p<0.001)$, with post hoc LSD com-

Table 2

Characteristics of stimuli across different accents (SD in brackets).

\begin{tabular}{llllll}
\hline Variable & Home & Yorkshire & Welsh & Italian & Polish \\
\hline Sentence duration & 1846.72 & 1965.51 & 1838.48 & 2153.80 & 1944.09 \\
$\quad(m s)$ & $(526.70)$ & $(542.02)$ & $(489.36)$ & $(496.77)$ & $(553.37)$ \\
Word duration (ms) & 526.70 & 542.02 & 489.36 & 496.77 & 553.37 \\
& $(88.16)$ & $(135.74)$ & $(76.96)$ & $(107.03)$ & $(82.06)$ \\
Phonological & 5.05 & 4.82 & 4.91 & 4.82 & 4.91 \\
$\quad$ uniqueness & $(1.10)$ & $(0.87)$ & $(1.00)$ & $(0.87)$ & $(1.00)$ \\
Phon. & 3.05 & 3.29 & 3.80 & 3.29 & 3.80 \\
$\quad$ neighbourhood & $(4.23)$ & $(4.64)$ & $(5.18)$ & $(4.64)$ & $(5.18)$ \\
Number of & 1.43 & 1.45 & 1.45 & 1.45 & 1.45 \\
$\quad$ morphemes & $(0.5)$ & $(0.5)$ & $(0.5)$ & $(0.5)$ & $(0.5)$ \\
Number of & 5.77 & 5.65 & 5.58 & 5.65 & 5.58 \\
$\quad$ phonemes & $(1.02)$ & $(1.05)$ & $(1.02)$ & $(1.05)$ & $(1.02)$ \\
Lexical frequency & 3.08 & 3.57 & $(3.80)$ & 3.57 & 3.80 \\
$\quad$ (subtlex) & $(1.31)$ & $(2.62)$ & $(2.97)$ & $(2.62)$ & $(2.97)$ \\
Phonological & 1.63 & 1.30 & 1.81 & 1.30 & 1.81 \\
$\quad$ levenshtein & $(3.47)$ & $(1.73)$ & $(3.43)$ & $(1.73)$ & $(3.43)$ \\
$\quad$ distance (PLD20) & & & & & \\
\hline
\end{tabular}


Table 3

Confusion matrix of accent identification for experiment stimuli; number of responses (proportion of responses).

\begin{tabular}{|c|c|c|c|c|c|c|}
\hline \multirow[t]{2}{*}{ Accent } & \multicolumn{6}{|l|}{ Identification } \\
\hline & South-West & Polish & Yorkshire & Italian & Welsh & Don't Know \\
\hline South-West & $421(.83)$ & $2(.00)$ & $35(.07)$ & $1(.00)$ & $14(.03)$ & $35(.07)$ \\
\hline Polish & $2(.01)$ & $147(.59)$ & $3(.01)$ & $92(.37)$ & $3(.01)$ & $3(.01)$ \\
\hline Yorkshire & $135(.54)$ & $5(.02)$ & $87(.35)$ & $1(.00)$ & $9(.04)$ & $15(.06)$ \\
\hline Italian & $3(.01)$ & $108(.43)$ & $9(.04)$ & $124(.49)$ & $3(.01)$ & $5(.02)$ \\
\hline Welsh & $22(.09)$ & $9(.04)$ & $27(.11)$ & $4(.02)$ & $192(.75)$ & $2(.00)$ \\
\hline
\end{tabular}

parisons showing that the identification of the home accent was significantly more accurate ( $83 \%$ correct) than foreign ( $54 \%$ correct, $p<0.001$ ) and regional accents (53\% correct, $p<0.001$ ), but that there was no significant difference in accuracy between the latter two categories $(F<1)$.

A repeated measures ANOVA established that perceived strength of accent differed significantly between accents $(F(4,76)=8.9, p<0.001)$. Fisher's LSD correction revealed that the South-West accent (rating of 2.6) and the Yorkshire accent (2.73) were significantly weaker than the Welsh $(3.43, p=0.004)$, the Polish (3.41, $p=0.004)$ and Italian (3.53, $p=0.003$ ) accents, with no other significant comparison.

In a similar test, a different set of 25 naïve South-West listeners rated the intelligibility of our stimuli by transcribing the final word of the sentences using a similar procedure to that used in the previous test. Words were reported as accurately transcribed if they matched the phonology of the word being produced (including homophones). Overall, word transcription accuracy was $88.4 \%$, with an ANOVA showing a main effect of accent $(F(2,48)=29.54$, $p<0.001$ ), with foreign accented sentences being less accurately transcribed $(80.60 \%)$ than regional accents $(90.71 \% ; t(24)=5.00$, $p<0.001)$ and home accent (93.83\%; $t(24)=8.25, p<0.001)$. There was no significant differences between the transcription of home and regional accents $(t(24)=1.73, p=0.09)$. The implications of this test will be commented upon in the discussion.

There follows a brief summary of the most salient characteristics of the accents recorded in this study, which are far from exhaustive, as accents consist of a wide range of subtle but noticeable variation that can defy concise written description.

\subsubsection{South West, UK (Home accent)}

The South West accent of English belongs to the family of Southern English accents (Wells, 1982), and thus has intonation patterns that do not depart significantly from that of the Received Pronunciation English. However, in the South West short vowels tend to be longer than in other South of England accents, especially in monosyllabic words in phrase-final or prominent position (Wells, 1982, p. 345). At the segmental level, it is distinct from RP English in its rhoticity, and the loss of the /æ/ and /a:/ distinction (Hughes \& Trudgill, 1996). Also words like 'boat' and 'gate' have usually retained their monophthong pronunciation (Wells, 1982).

\subsubsection{Leeds, Yorkshire, UK (Regional accent)}

The Leeds accent shares many features with other Northern English accents, including a tendency towards dropping the initial $/ \mathrm{h} /$ in unstressed words, replacing medial and terminal $/ \mathrm{t} /$ with a glottal stop, and a shortening of some vowels (British Library., 2009). Thus $/ \alpha$ :/ may be pronounced as $/ æ /$ and $/ \Lambda /$ is often pronounced as $/ v /$. Distinctive features of this accent include a reduction in the shaping of /ov/ such that it is pronounced like / $\mathbf{\varepsilon}: /$, and / $\mathrm{\partial} /$ is often pronounced as $/ \mathrm{v} /$, particularly in the middle of words such as 'without'. In our sample, the typical shortened vowels in words like gusto (pronounced /gustov/ rather than /g $\Lambda$ stəu/) are the main identifiable traits of this accent.

\subsubsection{South Wales, UK (Regional accent)}

According to Walters (2001), the South Wales accent is found to borrow many prosodic features from the Welsh language, resulting in a shortening of stressed vowels and lengthening of succeeding consonants, a pitch-rise from the stressed syllable and an increase in phonetic strength of the post-tonic syllables, and finally a shift of word stress from initial to penultimate or ultimate syllable in polysyllabic words. At the segmental level this accent is characterised by non-rhoticity (no post-vocalic "r"), the distribution of /æ/ and $/ \alpha$ :/ similar to that found in the North of England, and a slightly longer version of $/ æ /$ replacing the use of $/ \alpha$ : $/$ used in RP in words such as "bathtub" (Hughes \& Trudgill, 1996). Other features include the / $\varepsilon: /$ in "bird" being rounded to approach /ø:/, whilst the phoneme $/ 1 /$ is never velarised after a vowel.

\subsubsection{Italian (Foreign accent)}

Because Italian has only seven vowels (Adler, 1967) an Italian speaker will tend to project this reduced vowel space onto their English pronunciation. Words like 'lip' and 'leap' will tend to be pronounced in a similar fashion, with a medial vowel longer than that used in the RP pronunciation of 'lip'. Similarly, no distinction is made in Italian between the short / $/ \mathrm{d}$ and the long $/ \mathrm{u}: /$ (shortening words such as 'cooker'). There may also be confusion between $/ æ /$ and $/ \mathrm{e} /$, and also $/ \Lambda /$ and $/ æ /$. Schwa does not exist in Italian, leading to their replacement with clearly enunciated vowels and an associated interference in stress patterns (adding weight to the second syllable of 'dragon' for example).

In Italian $/ r /$ is frequently either rhotic or trilled, and so tends to result in words such as 'clover' pronounced with a final trilled $/ \mathrm{r} /$. In addition Italian does not contain the dental fricatives, $/ ð /$ and $/ \Theta /$. Therefore $/ \Theta /$ is often replaced with either $/ f /$ or $/ t /$, while $/ ð /$ is generally rendered as $/ d /$.

The contrast between the syllable-timed Italian and stresstimed English can result in more evenly stressed syllables and less reduction and elision than RP. These rhythmic and phonotactic differences largely account for increased sentence duration for Italian-accented stimuli.

\subsubsection{Polish (Foreign accent)}

Like Italian, Polish does not make use of the interdental fricatives, $/ ð /$ and $/ \Theta /$, and tends to render them as $/ v /$ or $/ d /$ and $/ f /$, /t/ or /s/ respectively (Gonet \& Pietroń, 2004). In addition, the reduced Polish vowel space of eight vowels can also result in vowel quality errors when producing English. Vowels may be incorrectly rhotacised, and schwa vowels may be over-articulated. There is also a notable confusion between / $\mathrm{r} /$ and /i:/, as in Italian, so that the word sick sounds very much like seek. Palato-alveolar consonants $\left(/ 3 /, / \mathrm{s} /, / d_{3} /\right.$ and $\left./ \mathrm{t} /\right)$ are also missing in Polish and may cause difficulties for some speakers, usually being articulated further forward, as alveolar or even interdental consonants. The English palato-alveolar / $\mathrm{r} /$ may be rhotic or trilled.

Polish has fixed stress on the penultimate syllable and reduction or elision is uncommon. This contrasts with RP, where stress is typically found on the penultimate or antepenultimate syllable (Cutler \& Carter, 1987), and stress-timing can shorten or elide 
unstressed syllables. This can result in incorrect stress patterns in Polish-accented English, with erroneously stressed syllables leading to over-articulation.

\subsection{Procedure}

A counterbalanced between and within participant design was used for this experiment with participants engaged in a go/no-go semantic categorisation task. Half of the participants heard sentences from the stimuli set 'HWP' (Home, Welsh, \& Polish speakers) and the other from set 'HYI' (Home, Yorkshire, \& Italian speakers). Sentences were presented in random order over headphones (Sennheiser HD580) using E-Prime software version 1.1 (Psychology Software Tools, 1996). At the start of each trial a white fixation cross was displayed on a black background with the sentence delivered after $600-800 \mathrm{~ms}$ ( $200 \mathrm{~ms}$ random jitter). Participants were instructed to press a button with their dominant hand only when the final word of the sentence was the name of an animal. 1700-1900 ms after the onset of the final word of the sentence the participants were given a visual cue to blink their eyes, which lasted for $1000 \mathrm{~ms}$. Participants were asked to blink their eyes each time they saw this symbol, and to avoid doing so at any other time. Trials were presented continuously, each separated by a $500 \mathrm{~ms}$ black screen, with a rest period provided half-way through the experiment. Each participant heard all 370 sentences from their stimulus set, 40 of which were semantic probe sentences, leaving 110 non-probe trials for each of the home, regional, and foreign accent categories.

\subsection{EEG recording and analysis}

Scalp voltages were collected from 30 actively amplified $\mathrm{Ag} /$ $\mathrm{AgCl}$ electrodes (actiCap, Brain Products $\mathrm{GmbH}$ ) mounted on an elastic cap (see Fig. 1). Eye movements and blinks were monitored using four additional electrodes attached below and lateral to the left and right eyes. EEG was referenced from a left mastoid electrode, and re-referenced offline to an average of left and right mas-

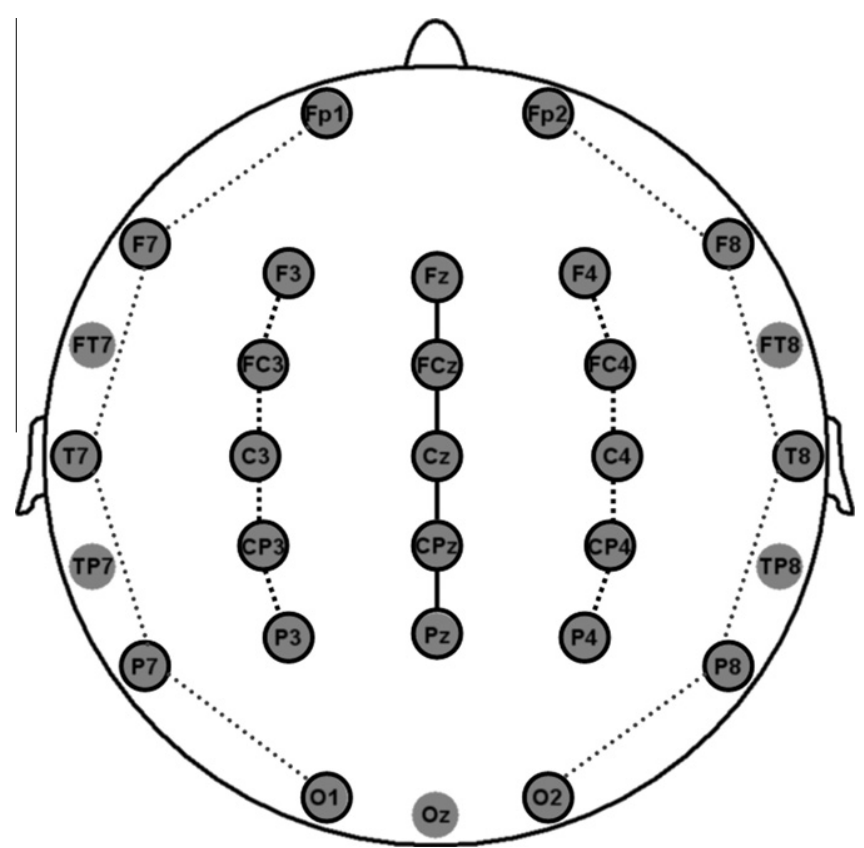

Fig. 1. Schematic of electrode montage (standard 10-20 system). Highlighted electrodes used during L1 (grey dotted column), L2 (black dashed column), and midline (solid black column) electrode analyses. toid activity, whilst an electrode positioned at AFz provided a ground. Inter-electrode impedances were maintained below $10 \mathrm{k}$ Ohm. Electrophysiological data was amplified using a Brain Vision BrainAmp MR Plus amplifier, continuously sampled at $500 \mathrm{~Hz}$ with an analogue band-pass filter of $0.01-250 \mathrm{~Hz}$ and offline digital band-pass filter of $0.1-40 \mathrm{~Hz}$. ERPs were calculated by averaging EEG time-locked to $100 \mathrm{~ms}$ before the onset of the final word of each sentence to $800 \mathrm{~ms}$ afterwards. Baseline correction was made using the $100 \mathrm{~ms}$ period prior to the onset of the final word, whilst direct current drift artifacts were removed using DC-detrend correction applied relative to the first and last $100 \mathrm{~ms}$ of the epoch. Following these corrections epochs contaminated by ocular or muscular artifact were rejected, as were those from the probe-trials and those with erroneous behavioural response. This resulted in the exclusion of $4.70 \%$ of the non-probe trials, with no significant difference in the distribution of these rejections over the three accent categories $(F<1)$. Separate ERPs were calculated for each electrode site, participant, and accent category. Mean amplitudes for two different time windows were analysed across three anterior-posterior columns of electrodes (shown in Fig. 1) using a separate ANOVA analysis for each column. The first of these consisted of the five midline electrodes Fz, FCz, $\mathrm{Cz}, \mathrm{CPz}$, and Pz. The $\mathrm{L} 1$ column consisted of five electrodes with different degrees of anterior/posterior position placed in both the left and right hemisphere of the outermost circumferential contour, consisting of FP1, FP2, F7, F8, T7, T8, P7, P8, 01 and O2. L2 consisted of a similar distribution to L1, but with electrodes following a circumferential contour closer to the midline, as follows: F3, F4, FC3, FC4, C3, C4, CP3, CP4, P3, and P4. These ANOVAs included within participant factors of accent (three factors: home, regional, and foreign), electrode anterior-posterior position (five electrodes) and hemisphere (apart from the midline column), and a between-participant factor of stimulus set (HWI vs. HYP). Only significant $(p<0.05)$ main effects and interactions with the factor of accent will be reported, appropriately adjusted using the Greenhouse and Geisser (1959) correction for the violation of sphericity.

\section{Results}

Grand Average ERPs time locked to the final word of sentences presented in home, regional, and foreign accent conditions can be seen in Fig. 2. Mean amplitude values for each of the conditions were calculated from $200-350 \mathrm{~ms}$ to $350-600 \mathrm{~ms}$, and can be seen in Figs. 3 and 4 respectively. This early epoch is intended to capture differences in phonological processing within the time-frame of the PMN, with the latter measures deviations within the N400 timeframe.

\subsection{Two-hundred to three-fifty milliseconds epoch}

Analyses revealed a main effect of accent in midline $(F(2,106)=12.33, p<0.001), \mathrm{L} 1(F(2,106)=5.62, p<0.005)$, and L2 $(F(2,106)=13.11, p<0.001)$ electrode columns. Subsequent pairwise comparisons between the three accents revealed significant differences between the regional and home accent conditions in midline $(F(1,53)=6.27, \quad p=0.015)$ and L2 $(F(1,53)=16.54$, $p<0.001)$ columns, between the foreign and home condition over midline $(F(1,53)=6.74, \quad p=0.012)$ and L2 $(F(1,53)=9.30$, $p<0.005)$, and also between the regional and foreign accents in midline $(F(1,53)=22.28, \quad p<0.001), \quad$ L1 $\quad(F(1,53)=15.24$, $p<0.001)$, and L2 $(F(1,53)=31.12, p<0.001)$.

The factor of accent was also found to interact with electrode position and hemisphere in column L1 $(F(8,424)=4.36$, $p<0.005)$. Planned comparisons of this three way interaction revealed significant $(p<0.05)$ differences between foreign and home 

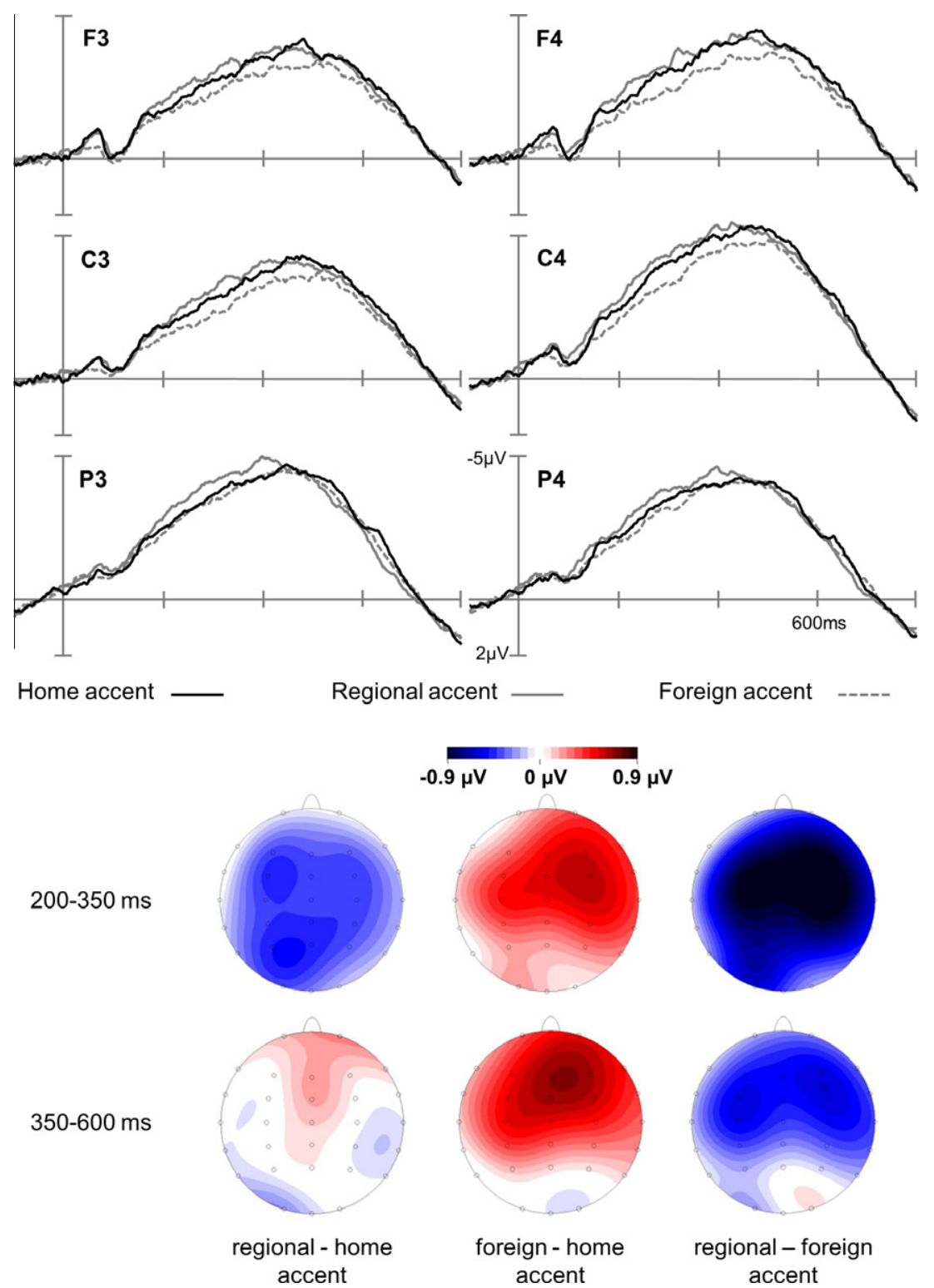

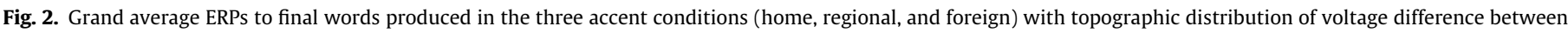
conditions between $200-350 \mathrm{~ms}$ and $350-600 \mathrm{~ms}$ after the onset of the final word.

conditions in right hemisphere electrodes FP2 $(F(1,53)=4.90)$, F8 $(F(1,53)=5.72)$, and T8 $(F(1,53)=5.72)$. There were also differences between foreign and regional conditions in right hemisphere electrodes FP2 $(F(1,53)=4.42)$, F8 $(F(1,53)=9.52)$, T8 $(F(1,53)=14.79)$, and P8 $(F(1,53)=14.95)$, and also in the left hemisphere electrode P7 $(F(1,53)=7.09)$. No significant differences were found between home and regional accents in any of the L1 electrodes $(F<2.30)$.

This meant that the amplitude of the negative-going PMN for regional accents was significantly greater than that of the baseline home accent condition, whilst the PMN for foreign accents was significantly less than that of the home accent.

\subsection{Three-fifty to six-hundred milliseconds epoch}

Analyses revealed a main effect of accent in midline $(F(2,106)=5.06, p<0.001), \mathrm{L} 1 \quad(F(2,106)=3.8, p=0.025)$, and L2 $(F(2,106)=6.69, p<0.005)$ electrode columns. Subsequent pairwise comparisons between the three accents showed no significant difference between home and regional accents in any electrode column $(F(1,53)<1)$. Foreign accents were found to be significantly different to the home accent over midline $(F(1,53)=9.55$, $p<0.005)$, L1 $(F(1,53)=5.38, p=0.024)$, and L2 $(F(1,53)=10.81$, $p<0.005)$, and different to regional accents across midline $(F(1,53)=4.64, p=0.036), \mathrm{L} 1 \quad(F(1,53)=5.51, p=0.023)$, and L2 $(F(1,53)=9.22, p<0.005)$ electrode columns.

The factor of accent was also found to interact with electrode anterior-posterior position in midline $(F(8,424)=3.38, p=0.033)$ and $\mathrm{L} 2(F(8,424)=3.60, p=0.023)$ columns. Planned comparisons of this interaction revealed significant $(p<0.005)$ differences between foreign accents and either home or regional accents in all midline electrodes apart from $\mathrm{Pz}(F<1)$, and all $\mathrm{L} 2$ bi-hemisphere electrode pairs apart from $\mathrm{O} 1$ and $\mathrm{O} 2(F<1)$. There were no significant differences between home and regional accent conditions in any of the electrode positions in either midline $(F<1.80)$ or L2 $(F<1)$.

This meant that the amplitude of the negative-going N400 for foreign accents was significantly less that of either the home or regional accent condition, with no significant difference in the N400 amplitude between regional and home accents. 

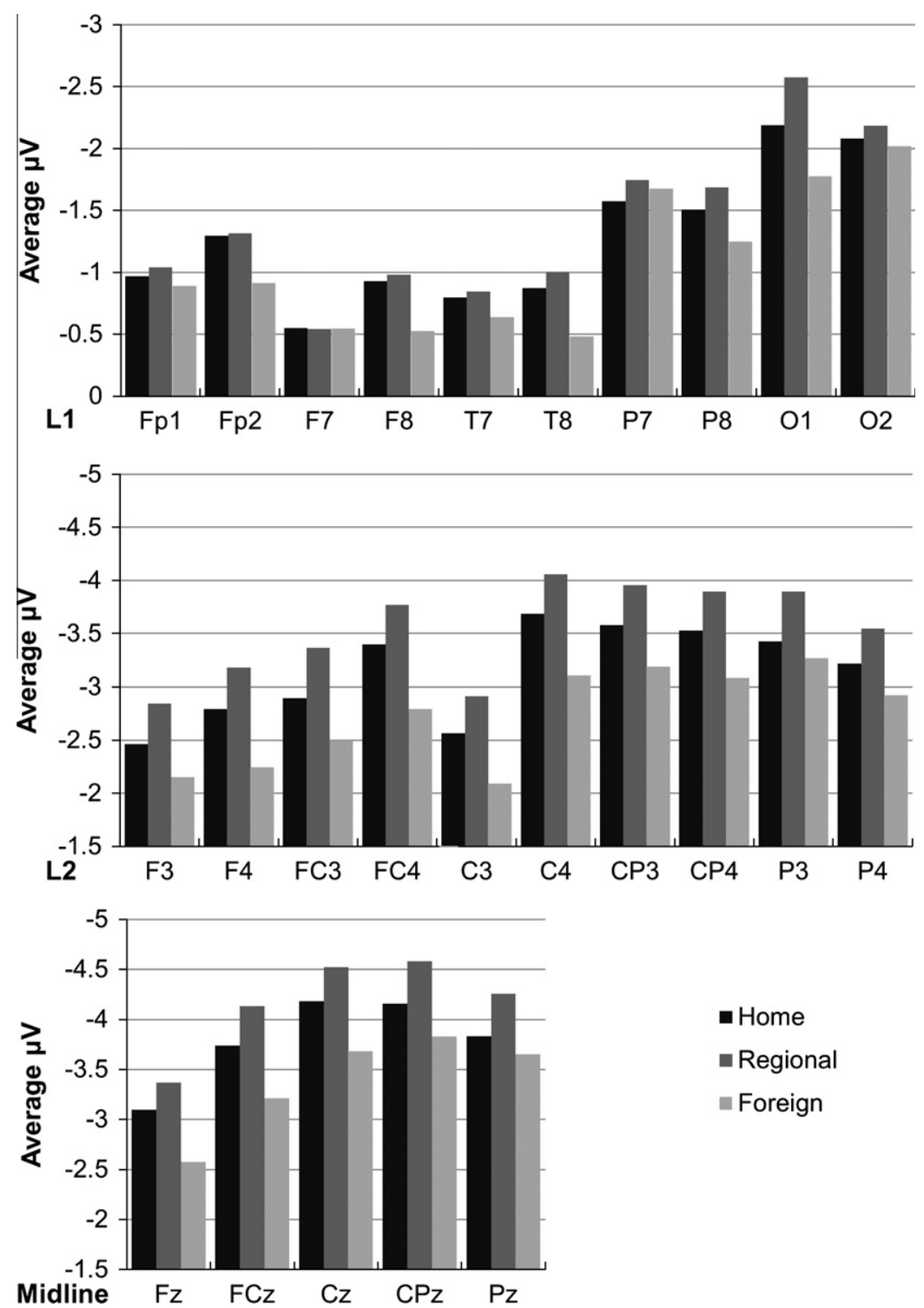

- Home

- Regional

a Foreign

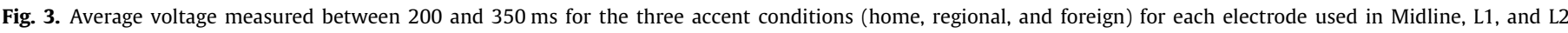
analyses.

\section{Discussion}

The main aim of this study was to establish whether the normalisation of foreign accents used the same cognitive architecture as that used for regional accents, or whether they required qualitatively different processing mechanisms. Using the participant's home accent as a neutral condition we compared ERPs recorded during the presentation of foreign and regional accented speech over time epochs designed to capture potential deviations in the PMN (200-350 ms) and N400 (350-600 ms) components. In the first epoch significant differences in average amplitudes indicated that the PMN was greater for regional than home accents. Conversely, foreign accents led to a significant reduction in the PMN when compared to home or regional accented speech. In the later epoch foreign accents elicited a significantly reduced N400 amplitude when compared to both other accent conditions, whilst there was no longer any significant difference in amplitude between regional and home accents.

The observation that the magnitude of the PMN was greater for regional accents than the home accent fits with the original predic- tions of both hypotheses, as shown in Table 1. However, the Perceptual Distance Hypothesis predicted that the PMN would increase still further with foreign accents, where we found was actually reduced in comparison with even the home accent. This is more consistent with the Different Processes hypothesis, where the unpredictable structure and variation inherent to foreign accented speech does not allow normalisation, leaving the accented-related "noise" intact. This could lead to a reduction in the PMN similar to that seen in low intelligible speech signals (e.g. Martin et al., 1997), or could even be analogous to superimposed noise within the framework of signal detection theory. It is well established that under severe levels of energetic masking, such as that produced by background speech or noise, listeners tend to rely less upon lexical-semantic knowledge and more on salient acoustic detail (see Mattys, Brooks, \& Cooke, 2009, for a review of the effects of energetic masking on speech processing). If the major problem when facing a foreign accent is the unreliability of acoustic/phonetic cues because of a degraded signal, it follows that listeners would be forced to give greater reliance upon topdown semantic knowledge to understand the incoming speech. 

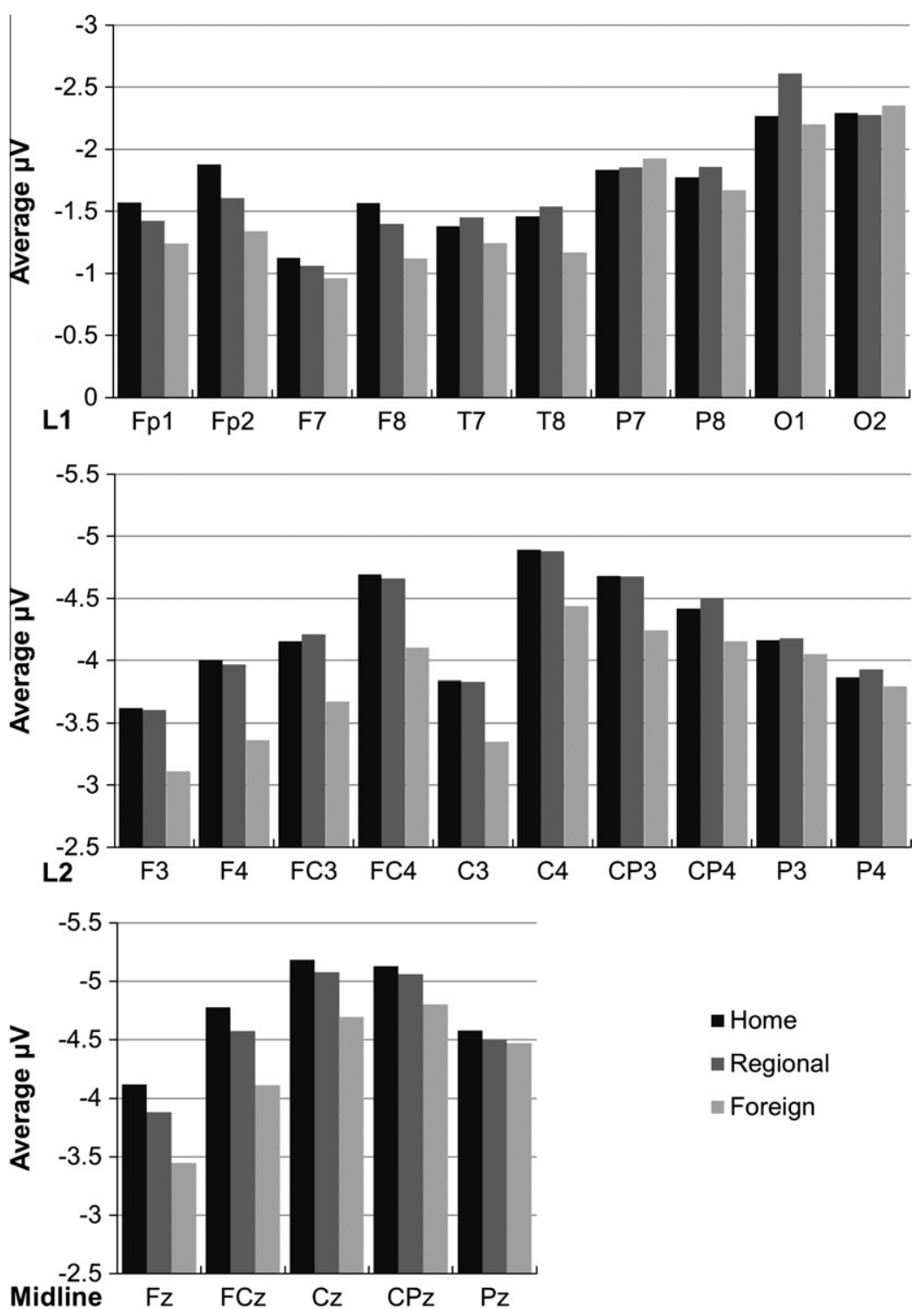

- Home

- Regional

a Foreign

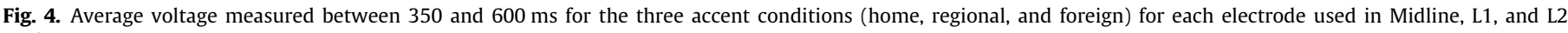
analyses.

In turn, this could offer an explanation for the reduction of the PMN amplitude in foreign compared to home accented speech. The unreliability of acoustic/phonetic cues and the switch to a top-down contextual-lexical knowledge might curtail listeners' reliance upon phonology, and thus their expectations with respect to this information.

This interpretation also raises the possibility that our findings are simply due to differences in intelligibility, with transcription accuracy found to be less accurate for foreign accented sentences $(80.60 \%)$, than those spoken with a regional accent $(90.71 \%)$, or the home accent (93.83\%). These differences were expected as previous studies also showed that foreign accents reduce intelligibility (e.g. Adank et al., 2009; Munro \& Derwing, 1995a), a feature that must be acknowledged as an inherent characteristic of these accents. However, whilst the gradation of intelligibility concurs with the Perceptual Distance Hypothesis, our electrophysiological results do not. If the deviations within the PMN simply reflected intelligibility then foreign accents would have greater amplitude than regional accents, with regional accents eliciting greater amplitude than the home accent. There remains the possibility that once intelligibility drops below a certain threshold this causes a step change in normalisation strategy reflected in the ERP. However, when Martin et al. (1997) examined the ERP responses to the contrast /ba/ versus /da/ in graded levels of noise masking they found no sudden changes in ERP as intelligibility reduced. Rather, they found progressive increases in latency and decrease in amplitude of ERP components (N1, N2, and P3; also see Polich, 1987, 2007 for the relation between P3 and the discriminability of a stimulus).

Whilst the polarity shift is the most salient difference between regional and foreign accents in the PMN epoch there were also differences in the topographic distribution of activity between these conditions. These were specific to the outermost circumferential contour of electrodes (L1), where activity for foreign accents would appear greater over right hemisphere anterior electrodes, and regional accents over left-hemisphere posterior and temporal electrodes. Only Adank, Noordzij, and Hagoort (2012) have previously sought to identify the neural locus of accent processing, proposing the involvement of the planum temporale (PT) based upon the functional model of spectro-temporal auditory processing by Griffiths and Warren (2002). Their functional magnetic res- 
onance imaging (fMRI) study used repetition suppression to compare activation in speaker and accent changes. They found that accent changes were predominantly associated with activation in left-lateralized posterior temporal areas, including PT, plus frontal areas including inferior frontal gyrus (IFG). Speaker switches on the other hand were shown to recruit predominantly right-lateralized activity including middle frontal gyrus and precuneus. To allow for both speaker and accent changes Adank et al. used a constructed novel accent using variations that were coherent to their speaker's original Dutch, and so could be considered as an artificial 'regional' accent. As can be seen there is a degree of overlap in the regions of activity between this and own regional accent condition, not obvious in the foreign accent condition, which could indicate a PT locus for accent normalisation specific to regional accents. However, this observation is highly speculative, given the limitations of low density EEG technique in identifying neural sources, compounded by the broad scalp differences seen between regional and foreign accent conditions.

In summary, our findings suggest that different strategies were adopted to process regional and foreign accents at a pre-lexical/ phonological level, lending support to the Different Processes Hypothesis. Furthermore, it would also appear that these earlier processing stages are sufficient to normalise unfamiliar regional accents, as by the 350-600 ms epoch there no longer any significant difference between the ERP for the home and regional accents. This is in contrast to foreign accented speech, where N400 amplitude was found to be significantly greater than that of the home and regional accents. This indicates that non-native phonological variations could not be fully normalised in pre-lexical processing levels, and so had a continued effect at lexical access and integration stages. These amplitude differences are similar to the findings of Aydelott et al. (2006) and Boulenger et al. (2011), who found reductions in the $\mathrm{N} 400$ when introducing noise or distortion to the final word of low cloze probability sentences. It was proposed that we would find a similar reduction, with the distortion provided by the acoustic/phonetic variability inherent in the foreign accents combined with the low cloze probability of the final words of our stimuli. Therefore, what we observe is a homothetic transformation of the signal generated by home accent speech, with a coefficient reflecting the amount of signal degradation. One might argue that this would support the Perceptual Distance Hypothesis, however it does not account for the effect of pre-lexical normalisation, which appears capable of reducing the 'signal degradation' caused by regional accents before semantic activation and integration. However, as pre-lexical normalisation would appear to be ineffective with foreign accented speech this necessitates the greater involvement of lexical processing, leading to the previously discussed N400 modulation.

Models of spoken word recognition make different claims regarding the locus of processing and representation of variations such as foreign or regional accents, however to our knowledge none has ever distinguished between the processing of these two types of accents. Recently McQueen, Cutler, and Norris (2006) have argued in favour of mixed models of spoken word recognition in which abstraction (as seen in models such as Pallier et al., 2001) would remain the core characteristic of the lexicon but with separate storage for speakers' specific features (as seen in proposals by Goldinger, 1998; Johnson, 1997; Klatt, 1979; Pierrehumbert, 2001, 2002) and also perhaps with storage for pronunciation variants, such as regional variants. Our results suggest a pre-lexical locus for such a storage device if they would be applied to regional accents, as the variation linked to this type of accent seems to be resolved at the lexical level. Whilst a pre-lexical deviant pronunciation storage device might prove useful for foreign accent processing, the variation between instances of accents would be problematic to the generation of pronunciation prototypes, and could explain the processing difficulties in foreign accents. The idea of accent-variant storage is also consistent with data from Sumner and Samuel (2009), who examined American English listeners with frequent exposure to a local variety of English (NewYork) and also a generic American accent. They found that all speakers could use either variant in immediate processing, but only those engaged with the local accent could encode both accents in long-term memory for later use (see also Ranbom \& Connine, 2007). This suggests that the consolidation of accent-related information in the accent-variant storage space varies as a function of exposure, or perhaps as a function of links between perception and production. It could also indicate that long-term encoding of accent variants is lexically based, challenging the idea of uniqueness of representations in the lexicon.

\subsection{Conclusions}

The results of this study provide new evidence indicating that regional and foreign accents recruit different normalisation mechanisms. It would appear that listeners are able to employ mechanisms at a pre-lexical processing level to normalise speech variation coherent to their own native language system, such that lexical processing appears relatively unperturbed by regional accents (see Sumner, 2011 for a similar argument). In contrast, the non-coherent variation inherent in foreign accents does not appear to be effectively normalised with unreliable phonetic/acoustic information forcing increasing reliance upon top-down contextual cues and reducing lexical activation.

\section{References}

Adank, P., \& McQueen, J. M. (2007). The effect of an unfamiliar regional accent on spoken word comprehension. In Proceedings of the XVIth international congress of phonetic sciences, Saarbrücken, Germany (pp. 1925-1928).

Adank, P., Evans, B. G., Stuart-Smith, J., \& Scott, S. K. (2009). Comprehension of familiar and unfamiliar native accents under adverse listening conditions. Journal of Experimental Psychology: Human Perception and Performance, 35(2), 520.

Adank, P., Noordzij, M. L., \& Hagoort, P. (2012). The role of planum temporale in processing accent variation in spoken language comprehension. Human Brain Mapping, 33(2), 360-372.

Adler, K. (1967). Phonetics and diction in singing: Italian, French, Spanish, German. Minneapolis: University of Minnesota Press.

Aydelott, J., Dick, F., \& Mills, D. L. (2006). Effects of acoustic distortion and semantic context on event-related potentials to spoken words. Psychophysiology, 43(5), 454-464.

Berman, S. M., Mandelkern, M. A., Phan, H., \& Zaidel, E. (2003). Complementary hemispheric specialization for word and accent detection. NeuroImage, 19(2), 319-331.

Best, C. T., McRoberts, G. W., \& Goodell, E. (2001). Discrimination of non-native consonant contrasts varying in perceptual assimilation to the listener's native phonological system. Journal of the Acoustical Society of America, 109, 775-794.

Boulenger, V., Hoen, M., Jacquier, C., \& Meunier, F. (2011). Interplay between acoustic/phonetic and semantic processes during spoken sentence comprehension: An ERP study. Brain and language, 116(2), 51-63.

Bradlow, A. R., \& Bent, T. (2003). Listener adaptation to foreign-accented English. In Proceedings of the XVth international congress of phonetic sciences (pp. 28812884).

British Library. (2009). Leeds. archival sound recordings. <http://sounds.bl.uk/ View.aspx?item=021M-C0900X08603X-0200V0.xml>.

Brunellière, A., Dufour, S., \& Nguyen, N. (2011). Regional differences in the listener's phonemic inventory affect semantic processing: A mismatch negativity (MMN) study. Brain and Language, 117(1), 45-51.

Brunellière, A., Dufour, S., Nguyen, N., \& Frauenfelder, U. H. (2009). Behavioral and electrophysiological evidence for the impact of regional variation on phoneme perception. Cognition, 111(3), 390-396.

Brysbaert, M., \& New, B. (2009). Moving beyond Kučera and Francis: A critical evaluation of current word frequency norms and the introduction of a new and improved word frequency measure for American English. Behavior Research Methods, 41(4), 977-990.

Chambers, J. K. (1992). Dialect acquisition. Language, 68(4), 673-705.

Chambers, J. K. (2002). Dynamics of dialect convergence. Journal of Sociolinguistics, 6(1), 117-130.

Clarke, C. M. (2000). Perceptual adjustment to foreign-accented English. Journal of the Acoustical Society of America, 107, 2856 
Clarke, C. M., \& Garrett, M. F. (2004). Rapid adaptation to foreign-accented English. Journal of the Acoustical Society of America, 116, 3647.

Clopper, C. G., \& Bradlow, A. R. (2009). Free classification of American English dialects by native and non-native listeners. Journal of Phonetics, 37(4), 436-451.

Clopper, C. G., \& Pisoni, D. B. (2004a). Some acoustic cues for the perceptual categorization of American English regional dialects. Journal of Phonetics, 32(1), 111-140.

Clopper, C. G., \& Pisoni, D. B. (2004b). Homebodies and army brats: Some effects of early linguistic experience and residential history on dialect categorization. Language Variation and Change, 16(01), 31-48.

Connolly, J. F., \& Phillips, N. A. (1994). Event-related potential components reflect phonological and semantic processing of the terminal word of spoken sentences. Journal of Cognitive Neuroscience, 6(3), 256-266.

Connolly, J. F., Phillips, N. A., Stewart, S. H., \& Brake, W. G. (1992). Event-related potential sensitivity to acoustic and semantic properties of terminal words in sentences. Brain and Language, 43(1), 1-18.

Conrey, B., Potts, G. F., \& Niedzielski, N. A. (2005). Effects of dialect on merger perception: ERP and behavioral correlates. Brain and Language, 95(3), 435-449.

Cutler, A., \& Carter, D. M. (1987). The predominance of strong initial syllables in the English vocabulary. Computer Speech E Language, 2(3-4), 133-142.

Cutler, A., Mehler, J., Norris, D., \& Segui, J. (1986). The syllable's differing role in the segmentation of French and English. Journal of Memory and Language, 25(4), $385-400$.

Davis, M. H., Johnsrude, I. S., Hervais-Adelman, A., Taylor, K., \& McGettigan, C. (2005). Lexical information drives perceptual learning of distorted speech: Evidence from the comprehension of noise-vocoded sentences. Journal of Experimental Psychology: General, 134(2), 222.

Derwing, T. M., Rossiter, M. J., Munro, M. J., \& Thomson, R. I. (2004). Second language fluency: Judgments on different tasks. Language Learning, 54(4), 655-679.

Desroches, A. S., Newman, R. L., \& Joanisse, M. F. (2009). Investigating the time course of spoken word recognition: Electrophysiological evidence for the influences of phonological similarity. Journal of Cognitive Neuroscience, 21(10), 1893-1906.

Dufour, S., Nguyen, N., \& Frauenfelder, U. H. (2007). The perception of phonemic contrasts in a non-native dialect. Acoustical Society of America Journal, 121, L131.

Dupoux, E., Pallier, C., Sebastian, N., \& Mehler, J. (1997). A destressing' deafness' in French? Journal of Memory and Language, 36, 406-421.

Eisner, F., \& McQueen, J. M. (2005). The specificity of perceptual learning in speech processing. Attention, Perception, \& Psychophysics, 67(2), 224-238.

Evans, B., \& Taylor, E. (2010). Investigating the time course of perceptual adaptation to unfamiliar accented speech. In M. G. Ashby, P. Ashby, \& R. A. Knight (Eds.), Proceedings of BAAP 2010.

Evans, B. G., \& Iverson, P. (2004). Vowel normalization for accent: An investigation of best exemplar locations in northern and southern British English sentences. Journal of the Acoustical Society of America, 115, 352.

Flege, J. E. (1988). Factors affecting degree of perceived foreign accent in English. Journal of the Acoustical Society of America, 84, 70-79.

Floccia, C., Butler, J., Goslin, J., \& Ellis, L. (2009). Regional and foreign accent processing in English: Can listeners adapt? Journal of Psycholinguistic Research, 38(4), 379-412.

Floccia, C., Goslin, J., Girard, F., \& Konopczynski, G. (2006). Does a regional accent perturb speech processing? Journal of Experimental Psychology: Human Perception and Performance, 32(5), 1276.

Girard, F., Floccia, C., \& Goslin, J. (2008). Perception and awareness of accents in young children. British Journal of Developmental Psychology, 26(3), 409-433.

Goldinger, S. D. (1998). Echoes of echoes? An episodic theory of lexical access. Psychological Review, 105(2), 251-279.

Gonet, W., \& Pietroń, G. (2004). The Polish tongue in the English ear. Zeszyty Naukowe Państwowej Wyszej Szkoły Zawodowej w Koninie, 4(1), 56-65.

Greenhouse, S. W., \& Geisser, S. (1959). On methods in the analysis of profile data. Psychometrika, 24(2), 95-112.

Griffiths, T. D., \& Warren, J. D. (2002). The planum temporale as a computational hub. Trends in Neurosciences, 25(7), 348-353.

Hagoort, P., \& Brown, C. M. (2000). ERP effects of listening to speech: Semantic ERP effects. Neuropsychologia, 38(11), 1518-1530.

Hervais-Adelman, A., Davis, M. H., Johnsrude, I. S., \& Carlyon, R. P. (2008). Perceptual learning of noise vocoded words: Effects of feedback and lexicality. Journal of Experimental Psychology: Human Perception and Performance, 34(2), 460.

Hughes, A., \& Trudgill, P. (1996). English accents and dialects: An introduction to social and regional varieties of English in the British Isles (vol. 3). London: Arnold.

Johnson, K. (1997). Speech perception without speaker normalization: An exemplar model. In K. Johnson \& J. Mullenix (Eds.), Talker variability in speech processing (pp. 145-165). San Diego: Academic Press.

Jongman, A., Wade, T., \& Sereno, J. (2003). On improving the perception of foreignaccented speech. In Proceedings of the 15th international congress of phonetic sciences (pp. 1561-1564).

Klatt, D. H. (1979). Speech perception: A model of acoustic-phonetic analysis and lexical access. Journal of Phonetics, 7, 279-312.

Kutas, M., \& Hillyard, S. A. (1984). Brain potentials during reading reflect word expectancy and semantic association. Nature, 307(5947), 161-163.

Lane, H. (1963). Foreign accent and speech distortion. Journal of the Acoustical Society of America, 35, 451.
Luce, P. A., \& McLennan, C. T. (2005). Spoken word recognition: The challenge of variation. In The handbook of speech, perception (pp. 590-609).

Marslen-Wilson, W. D. (1987). Functional parallelism in spoken word-recognition. Cognition, 25(1), 71-102.

Martin, B. A., Sigal, A., Kurtzberg D., \& Stapells, D. R. (1997). The effects of decreased audibility produced by high-pass noise masking on cortical event-related potentials to speech sounds/ba/and/da. Journal of the Acoustical Society of America, 101(3), 1585-1599.

Mattys, S. L., Brooks, J., \& Cooke, M. (2009). Recognizing speech under a processing load: Dissociating energetic from informational factors. Cognitive Psychology, 59(3), 203-243.

Maye, J., Aslin, R. N., \& Tanenhaus, M. K. (2008). The weckud wetch of the wast: Lexical adaptation to a novel accent. Cognitive Science, 32(3), 543-562.

McClelland, J. L., \& Elman, J. L. (1986). The TRACE model of speech perception. Cognitive Psychology, 18(1), 1-86.

McQueen, J. M., Cutler, A., \& Norris, D. (2006). Phonological abstraction in the mental lexicon. Cognitive Science, 30(6), 1113-1126.

Munro, M. J., \& Derwing, T. M. (1995a). Processing time, accent, and comprehensibility in the perception of native and foreign-accented speech. Language and Speech, 38(3), 289-306.

Munro, M. J., \& Derwing, T. M. (1995b). Foreign accent, comprehensibility, and intelligibility in the speech of second language learners. Language Learning, 45(1), 73-97.

Naatanen, R., Lehtokoski, A., Lennes, M., Cheour, M., Huotilainen, M., Iivonen, A. et al. (1997). Language-specific phoneme representations revealed by electric and magnetic brain responses. Nature, 385(6615), 432-434.

Newman, R. L., \& Connolly, J. F. (2009). Electrophysiological markers of pre-lexical speech processing: Evidence for bottom-up and top-down effects on spoken word processing. Biological Psychology, 80(1), 114-121.

Newman, R. L., Connolly, J. F., \& McIvor, K. (2003). Influence of phonological expectations during a phoneme deletion task: Evidence from event-related brain potentials. Psychophysiology, 40(4), 640-647.

Norris, D. (1994). Shortlist: A connectionist model of continuous speech recognition. Cognition, 52(3), 189-234.

Norris, D., McQueen, J. M., \& Cutler, A. (2003). Perceptual learning in speech. Cognitive Psychology, 47(2), 204-238.

Pallier, C., Colomé, A., \& Sebastián-Gallés, N. (2001). The influence of nativelanguage phonology on lexical access: Exemplar-based versus abstract lexical entries. Psychological Science, 12(6), 445-449.

Pallier, C., Sebastian-Gallés, N., Dupoux, E., Christophe, A., \& Mehler, J. (1998). Perceptual adjustment to time-compressed speech: A cross-linguistic study. Memory \& Cognition, 26(4), 844-851.

Pierrehumbert, J. B. (2001). Exemplar dynamics: Word frequency, lenition and contrast. In J. Bybee \& P. Hopper (Eds.). Frequency and the emergence of linguistic structure (Vol. 45, pp. 137-157). Amsterdam: John Benjamins.

Pierrehumbert, J. B. (2002). Word-specific phonetics. In C. Gussenhoven \& N Warner (Eds.). Laboratory phonology (Vol. 7, pp. 101-139). Berlin: Mouton de Gruyter.

Pinet, M., Iverson, P., \& Evans, B. G. (2011). Perceptual adaptation for L1 and L2 accents in noise by monolingual British English listeners.

Polich, J. (1987). Task difficulty, probability, and inter-stimulus interval as determinants of P300 from auditory stimuli. Electroencephalography and Clinical Neurophysiology/Evoked Potentials Section, 68(4), 311-320.

Polich, J. (2007). Updating P300: An integrative theory of P3a and P3b. Clinical Neurophysiology, 118(10), 2128-2148.

Ranbom, L. J., \& Connine, C. M. (2007). Lexical representation of phonological variation in spoken word recognition. Journal of Memory and Language, 57(2), 273-298.

Rivera-Gaxiola, M., Csibra, G., Johnson, M. H., \& Karmiloff-Smith, A. (2000) Electrophysiological correlates of cross-linguistic speech perception in native English speakers. Behavioural Brain Research, 111(1-2), 13-23.

Samuel, A. G., \& Kraljic, T. (2009). Perceptual learning for speech. Attention, Perception, \& Psychophysics, 71(6), 1207-1218.

Schmid, P. M., \& Yeni-Komshian, G. H. (1999). The effects of speaker accent and target predictability on perception of mispronunciations. Journal of Speech, Language, and Hearing Research, 42(1), 56-64.

Sebastián-Gallés, N., Vera-Constán, F., Larsson, J. P., Costa, A., \& Deco, G. (2009) Lexical plasticity in early bilinguals does not alter phoneme categories: II. Experimental Evidence. Journal of Cognitive Neuroscience, 21(12), 2343-2357.

Shestakova, A., Brattico, E., Soloviev, A., Klucharev, V., \& Huotilainen, M. (2004). Orderly cortical representation of vowel categories presented by multiple exemplars. Cognitive Brain Research, 21(3), 342-350.

Sumner, M. (2011). The role of variation in the perception of accented speech Cognition, 119(1), 131-136.

Sumner, M., \& Samuel, A. G. (2009). The effect of experience on the perception and representation of dialect variants. Journal of Memory and Language, 60(4), 487-501.

Walters, J. R. (2001). English in Wales and a 'Welsh Valleys' accent. World Englishes, 20(3), 285-304

Wells, J. C. (1982). Accents of English: An introduction. Cambridge: Cambridge University Press. 